\title{
AN IMMERSIVE VIRTUAL ENVIRONMENT FOR REVIEWING MODEL-CENTRIC DESIGNS
}

\author{
Romero, Victor; \\ Pinquié, Romain; \\ Noël, Frédéric \\ Univ. Grenoble Alpes, CNRS, Grenoble INP, G-SCOP, 38000 Grenoble, France
}

\begin{abstract}
New design objectives as the digital twin encourage companies to replace the tradition documentbased systems engineering approach by a model-centric one. All views of the system rely on different types of models that serve many objectives, especially to improve communication among stakeholders. However, the increasing number of heterogeneous models jeopardize communication at the end. Indeed, to get a holistic view of the virtual definition, engineers have no other alternative than to navigate through numerous models requiring domain-specific software and language. In this paper, we propose to use virtual reality to develop an immersive environment for a collaborative modelcentric review of engineered systems. The virtual environment, which relies on a digital thread stored in a graph-oriented database, enables users to explore a model-centric design by navigating through the models in a unique virtual space. To illustrate our proposal, we use a model-centric design of a telescope and shows how our preliminary prototype supports the reviewing activity with data limited to the architecture and geometry. Future works will concentrate on the integration of data related to other perspectives on the system.
\end{abstract}

Keywords: Virtual reality, Systems Engineering (SE), Product modelling / models, Design review, Immersion

\section{Contact:}

Romero, Victor

Université Grenoble Alpes

G-SCOP

France

victor.romero@grenoble-inp.fr

Cite this article: Romero, V., Pinquié, R., Noël, F. (2021) 'An Immersive Virtual Environment for Reviewing ModelCentric Designs', in Proceedings of the International Conference on Engineering Design (ICED21), Gothenburg, Sweden, 16-20 August 2021. DOI:10.1017/pds.2021.45 


\section{INTRODUCTION}

\subsection{Context}

The recent paradigm shift from document-centric to model-centric systems engineering provides a new opportunity to adopt a parsimonious approach that enables engineers to develop and manage technological systems more efficiently, especially by minimising communication misunderstandings. Indeed, in model-centric systems engineering, the communication among various stakeholders with different background is facilitated by an overarching digital approach that use models to formalise specific views on the system-of-interest. Thus, architects start by defining operational scenarios using static diagrams (e.g., SysML use case diagram, SysML block definition diagram, user stories, goaloriented diagram, value models, etc.) and dynamic diagrams (e.g., Simulink models) to describe how the system will be used and in which context. Then, they specify model-based requirements (e.g., SysML requirement diagrams, logical predicates, state machines, etc.) which are subsequently implemented by all sorts of models depicting the numerous views of the system architecture (e.g., SysML internal block diagram, SysML activity diagram, 3D skeleton and allocated volumes, etc.). After reaching a sufficient level of details, experts focus on the modelling of structural and behavioural properties using new models including, but not limited to, Computer-Aided Design (CAD), Finite Elements Analysis (FEA), kinematic, dynamic models. In parallel, the cross-functional department concentrates on the safety view using other types of models (e.g., failure modes and effects tables, fault trees, reliability block diagrams, failure diagrams, etc.). By stepping back from the current model-centric approach, we observe that emerging factors negatively influence the communication among the stakeholders, especially for collaboratively reviewing a design.

\subsection{Problem}

Our experience with companies willing to deploy a model-centric system engineering approach has shown that communication among stakeholders is facing new challenges. Indeed, the increasing number of models makes it difficult to understand the system and increases the difficulty to manage their configurations. Moreover, it is impossible to obtain a holistic view because models come from different software. A pragmatic way to combine models that belong to a different view - e.g., functional, and physical - is to take screenshots of models before inserting them next to each other in a document or opening two windows or using two screens. What if we need to simultaneously access many views? Even when we concentrate on a specific SysML software to model the architecture of a system, the architect has no other alternative to navigate either through a bewildering array of diagrams or tens of windows to get the gist of the architecture and must reconstruct implicit links between the elements while navigating. Furthermore, in a model-centric approach, the interpretation of models requires stakeholders to learn domain-specific languages limiting the ability of a person to interact with colleagues who have a different view on the system leading to the silo effect. Finally, we observe that many companies use SysML to define the architecture of systems and that the influence of software engineering - SysML being a UML profile tailored for systems engineering - lead to undesired effects, such as an inflexible management of multiple views on a single element (Herzog, Pandikow and Ab, 2005).

With the increasing complexity of the models produced, the traditional design review that occurs in a meeting where people join with PowerPoint slides, which contain screenshots of models leading to the loss of the original visual and interactive capabilities, is no further adapted to the development and management of engineered systems.

\subsection{Proposal}

Because of the problems previously discussed, there is a growing sense that the traditional practices for reviewing a design have ceased to function adequately since the adoption of a model-centric design approach and this observation motivates us to ask the research question:

\section{Research Question:}

\section{Does immersion through virtual reality techniques facilitate model-centric design reviews?}


In the field of mechanical engineering, the transition from paper-based blueprints to 2D digital blueprints before being extended to $3 \mathrm{D}$ in the model-based definition shows that $3 \mathrm{D}$ makes the technical information more accessible to people. Moreover, the development of virtual reality encouraged designers to review digital mock-up in a collaborative and immersive virtual environment (such as a CAVE) rather than to use traditional 2D blueprints and 3D models. This evolution and similar ones (e.g. the use of immersive environment for the visualisation of big data - immersive visual analytics (Chandler et al., 2015)) encourage us to formulate the research hypothesis:

\section{Research Hypothesis:}

\section{We assume that the unification of models in a unique and immersive virtual environment provides a holistic bird's-eye-view that facilitates model-centric design review.}

In the next section, we will review the existing literature that encouraged us to assume that virtual reality is a promising technology to ease the review of a model-centric design.

\section{LITERATURE REVIEW}

If virtual reality has been largely studied in recent years, it is because it offers undeniable advantages for analysing. This has already been demonstrated in several application domains as medical (Mirhosseini et al., 2014), ergonomics (Michalos et al., 2018), or archaeology (Bennett, Zielinski and Kopper, 2014). For engineering activities, virtual reality, for instance, is used for maintenance scenarios (Louison et al., 2017) and behavioural simulation (Waurich and Weber, 2017), which are two user-centric applications. Although all these domains contain some elements motivating us to assume that virtual reality would facilitate the review of a model-centric design, this literature review concentrates on two applications that are tightly related to our domain of interest: the review of mechanical designs and software architecture.

\subsection{Virtual Reality for the Design Reviews of Mechanical Designs}

An immersive visualisation implies a better appreciation of the shapes, sizes, and colours of the designed system leading to several applications in mechanical engineering. For instance, virtual reality has been widely used for reviewing the design of CAD assemblies, especially for appreciating access to maintenance areas (Louison et al., 2017). Virtual reality is so popular in CAD that all main CAD software editors provide a pipeline to switch from native desktop visualisation to an immersive environment (e.g., SolidWorks ${ }^{1}$ or CATIA $^{2}$ ). Academic studies also demonstrate the benefits to use an immersive virtual environment for CAD reviews, especially for adding features as annotation or objects selection (Aromaa et al., 2012; de Casenave and Lugo, 2018). More recently, applications such as the MeetinVR of Varjo ${ }^{3}$ and StayAtHome developed by the research lab of Siemens ${ }^{4}$, demonstrate that the need for collaboration in such an immersive environment is more and more important and, especially with the COVID crisis. Although virtual reality turns out to be a technology of interest for supporting design reviews in mechanical engineering, we observe that existing applications are limited to the review of CAD models, whereas model-centric design requires the review of many other types of models.

\subsection{Virtual Reality for the Design Review in Software Engineering}

The mushrooming of models is an old problem in software engineering. Indeed, to represent the architecture of a software, developers use the UML language and, for complex software, the number of models supporting the architecture starts becoming too important to be understood or analysed during project reviews (Maletic, Leigh and Marcus, 2001). To enable scalability, software engineers tried to display the elements of an architecture (hierarchical structure of components, relationships between

1 https://www.solidworks.com/fr/partner-product/demo3dvr-solidworks (Accessed 2020-12-11)

$2 \mathrm{https}: / /$ www.3ds.com/stories/never-blind-in-vr/ (Accessed 2020-12-11)

$3 \mathrm{https}: / /$ varjo.com/solutions/design-and-engineering/ (Accessed 2020-12-11)

4 https://new.siemens.com/global/en/company/stories/research-technologies/digitaltwin/virtual-sim-lab.html (Accessed 2020-12-11) 
components, quality metrics of codes, UML diagrams, etc.) and of the source code (functions, classes, packages, etc.) in an immersive environment using non-expert visual metaphors such as islands (Schreiber and Misiak, 2018), cities (Oberhauser and Lecon, 2017), and a solar system (Averbukh et al., 2019). Stakeholders are then able to organise collaborative reviews or simply analyse the structure of the code alone in the environment. The design of complex systems produces a huge number of models but not all of them are in 3D. Indeed, there are many engineering abstract data, especially at the beginning of the design process. These examples from software engineering demonstrate that it is possible and may be relevant to represent abstract data with $3 \mathrm{D}$ visual metaphors.

From this literature review, we can draw two conclusions on the current state of the art. First, academic studies and industrial applications have shown that virtual reality provides engineers with advanced visualisation and interaction capabilities for reviewing 3D models in mechanical engineering, but also in software engineering when 3D metaphors replace abstract representations. Second, no one has attempted to use virtual reality to support the review of a model-centric design to provide a holistic view of models. Thus, in the following sections, we will describe our virtual environment for a collaborative modelcentric design review before illustrating it on the design of a GO-TO telescope.

\section{IMMERSIVE VIRTUAL ENVIRONMENT FOR A COLLABORATIVE MODEL- CENTRIC DESIGN REVIEW}

In this section, we will describe the specifications and the preliminary design of a virtual environment.

\subsection{Description}

[Mission] To facilitate the review of a design based on a model-centric approach, we suggest using virtual reality to develop an immersive virtual environment that unifies all perspectives (Figure 1). Reviewing the virtual definition in a unique space with advanced visualisations and interactions should enable stakeholders to naturally explore related data in different views of the system by navigating through the models' interdependencies. Models interdependencies are linked data in a graph-oriented database fed by native models through standardised exchange formats, software APIs, and ad-hoc connectors.

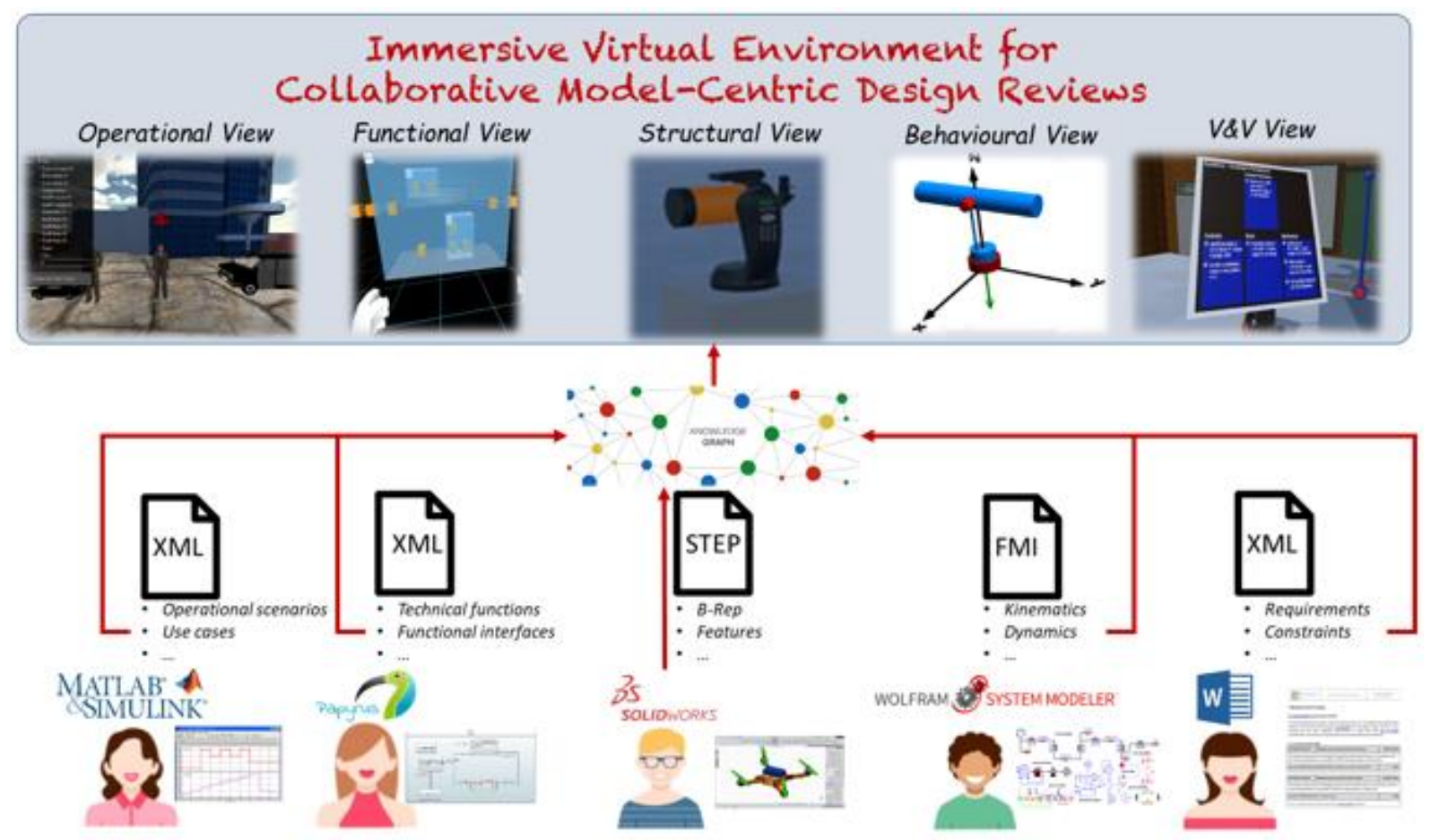

Figure 1. Immersive virtual environment for model-centric design reviews

[Stakeholders] The virtual environment is designed to be used by anyone. From experts in engineering to the system's architects, to the cross-functional functions (e.g. marketing, safety, quality, 
etc.), everyone shall be able to use the virtual environment without requiring intensive training. Indeed, unlike model and simulation environments that rely upon domain-specific languages, virtual reality benefits from natural interactions and visual metaphors. Blueprints in mechanical engineering is perhaps the most relevant example since the interpretation of $2 \mathrm{D}$ technical drawings requiring the learning of a technical language have been replaced by 3D representations and annotations in immersive virtual design review environments.

[Services] The services the virtual environment provides to the stakeholders derive from the main goals of design reviews in model-centric systems engineering.

- To understand the design: The virtual environment enables stakeholders to have a better understanding of the virtual definition of the system by quickly navigating through the models without the need to switch from one software to another, by visualising the link between the data produced and visualising the 3D model as it is already commonly done (Chen, Zucco and Olechowski, 2019), which helps to understand the system designed. Moreover, visual metaphors facilitate the reading of models that require the learning of a specific syntax and semantic. Architecture models such as SysML and Capella also require navigation through several diagrams in different windows before having a global understanding of the design, whereas the large field of view in virtual reality and head tracking capabilities lead to a seamless exploration.

- To verify the quality of models: Being able to unify models in a single environment is the opportunity to make sure that the virtual definition of the system is complete and correct. On the one hand, verifying the completeness is making sure there is a continuity in the digital information e.g., a requirement derives from a need, a CAD part satisfies one or several requirements, the mechanical interfaces in a SysML internal block diagram are equivalent to the ones in the CAD assembly, etc. The implementation of linked data, which is also known as digital thread (Hedberg, 2018), provides an integrated information flow in a knowledge graph (Huet et al., 2020) that connects all the design concepts stored in native models. On the other hand, checking the correctness is making sure the model does not contain design or modelling errors. The collaboration of people with a different background, the unification of models and the advanced visualisation capabilities logically increase the likelihood to detect an error in the models.

- To control the impact of changes: The analysis of impacts due to a change in the virtual definition of a system is a challenging task when models are independent. Integrating models in a single environment enables us to see how a change vertically (top-down and bottom-up) propagates through the systemic levels and horizontally affects colleagues' view at a given systemic level.

- To capture the knowledge produced by the exchanges: The tracking capabilities of virtual reality technologies enable the virtual environment to collect and analyse the exchanges between the stakeholders. Techniques allow then to transform these exchanges into knowledge (Dai and Velde, 2017), reusable by the company in the future.

\subsection{Implementation}

For the immersive environment that we previously introduced, we developed the first version of a prototype. Studies show that is possible to represent the data produced by the design with a graph (Bajaj and Hedberg, 2018) and that links between nodes of the graph from different models are known as digital threads (Hedberg, 2018). Our application follows the same strategy of linked data using the graph-oriented database $\mathrm{NeO}^{\mathrm{j}} \mathrm{j}^{5}$.

The nodes of the graph serve to access data in engineering models (function, requirement, kinematic and dynamic behaviour, CAD part, CAD assembly, failure cause, failure symptoms, etc.) and the relationships serve to link the data. The data produced in the engineering software (Papyrus for SysML and CATIA for CAD in our use case) are exported in a standardised format before being parsed by a C\# script and stored in the graph. Note that, the approach is not limited to any software as we use standardised exchange formats. The immersive environment is developed with the rendering engine Unity. To connect the knowledge graph to the rendering engine, we use the DLL provided by Neo4j that enables us to access the graph with a C\# script and network communication. C\# scripting also serves to display the relevant information and create interactions with the user. Finally, since we used

5 https://neo4j.com/ (Accessed 2020-12-11) 
the SteamVR plug-in for Unity 3D, the application can run on any SteamVR compatible device (HTC Vive Pro, Valve Index, etc.).

Table 1. List of technologies used to develop the prototype

\begin{tabular}{|l|l|}
\hline \multicolumn{2}{|c|}{ Software } \\
\hline \multirow{2}{*}{ Rendering engine } & Unity 3D \\
\cline { 2 - 3 } & C\# programming \\
\hline \hline Database & Neo4j \\
\hline \multirow{2}{*}{ Models production } & CATIA v5 (3D model) \\
\cline { 2 - 2 } & Papyrus (Architecture model) \\
\hline & Hardware \\
\hline Visualisation & HMD (Vive Pro or Valve Index) \\
\hline Interactions & HMD Controllers (Vive Pro or Valve Index) \\
\hline
\end{tabular}

\section{USE CASE: IMMERSIVE DESIGN REVIEW OF A TELESCOPE}

To illustrate our proposal, we use a model-centric design of a GO-TO telescope from Celestron ${ }^{6}$ and show how our preliminary prototype for the immersive virtual environment supports the reviewing activity. Since this is the beginning of our research, we limit the data to the architecture view and geometry view. In the close future, more models will be added to our study. Note that the appreciation of the benefit may be limited at first glance, but the combination of the two views is already a step towards our objective of providing a holistic view on heterogeneous and scattered models.

A GO-TO telescope is a telescope that can automatically find astronomical objects registered in a database. It is composed of two main subsystems: the optical subsystem to visualise astronomical objects and the support subsystem to move the optical subsystem.

\subsection{Model-Based Design}

Our use case starts with the model-centric design of the telescope with a focus on the architecture and the CAD models. The architecture of the studied system is largely inspired by the work of Mhenni et al. (2014). To build the models, we use the Papyrus plug-in of Eclipse. Papyrus is an open-source software, makes access to data easier. For each system (or sub-system), we defined its stakeholders, associated functions and requirements, and the logical architecture. All these elements are spread in several SysML models. All these data are then stored in a graph database, following the structure described in Figure 2. The 3D model of the telescope is designed with CATIA. The data contained in the model produced can be extracted thanks to macros and STEP file and stored in the same database as the architecture model. The 3D model, which is used for the visualisation, is transformed into an OBJ format to be imported into the virtual environment.

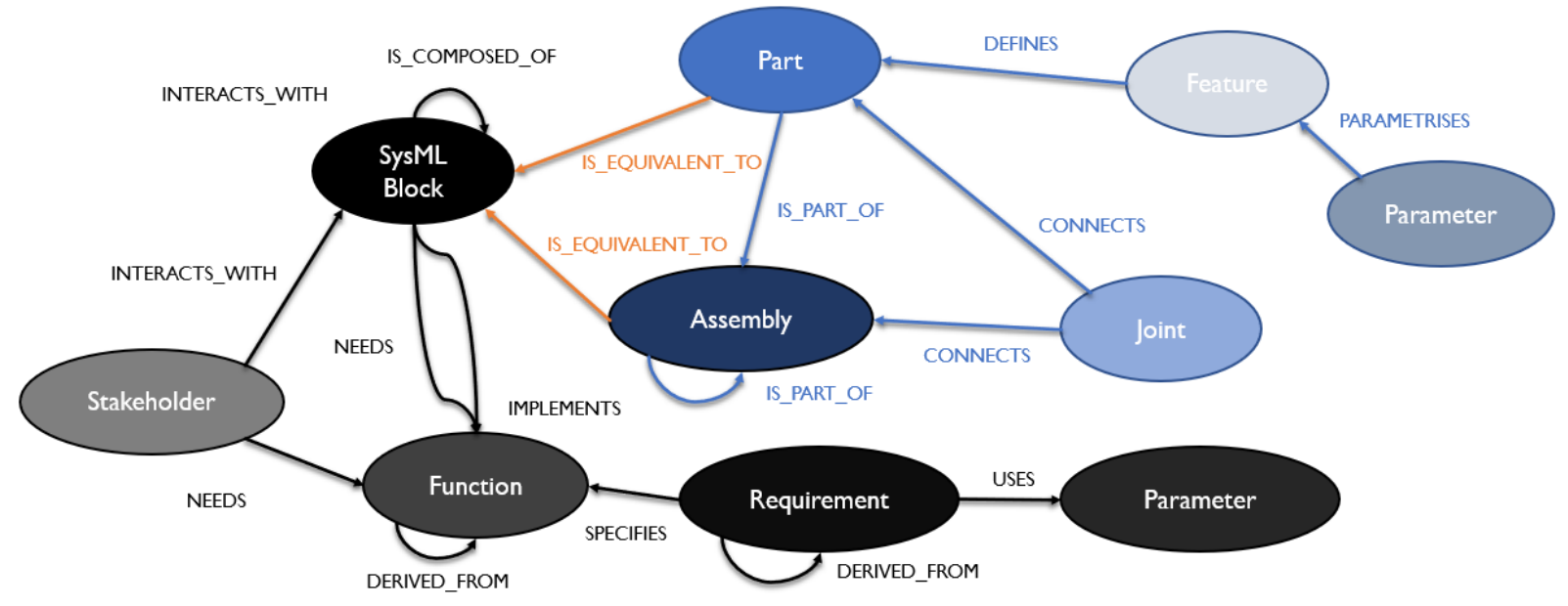

Figure 2. Minimum ontology for design review 


\subsection{Immersive and Interactive Metaphors}

With this single use case, we can imagine several design review scenarios. In this paper, we focus particularly on maintaining the coherence between all models. The goal of the environment is then to offer a display of different models simultaneously to enable the user to compare them or to have a better understanding by navigating through the information stored in the models and visualising the links between all the elements of the database. Before developing a virtual scene that supports this operational scenario, we shall specify the visual and interactive metaphors.

\subsubsection{Visualisation}

\section{An enriched 3D visualisation}

To specify the visualisation the environment should provide, we try to answer three questions: What we want to visualise, why we want this visualisation, and how the environment may provide this view.

Table 2. Specification of the 3D enriched view

\begin{tabular}{|l|l|l|}
\hline What? & Why? & How? \\
\hline $\begin{array}{l}\text { A 3D view of the system on } \\
\text { which we add information } \\
\text { provided by other models } \\
\text { (Architecture model in this first }\end{array}$ & $\begin{array}{l}\text { of have a comprehensible view } \\
\text { of the global system (visualising }\end{array}$ & $\begin{array}{l}\text { Querying a database to display } \\
\text { relevant information }\end{array}$ \\
\cline { 2 - 3 } environment) & $\begin{array}{l}\text { To have an intuitive view of the } \\
\text { relationships linking the data. }\end{array}$ & $\begin{array}{l}\text { Querying a database to display } \\
\text { the relevant relationships }\end{array}$ \\
\hline & $\begin{array}{l}\text { Providing information in } \\
\text { function of the distance to an } \\
\text { object, or by voice control }\end{array}$ \\
\cline { 3 - 4 } & $\begin{array}{l}\text { Displaying information in } \\
\text { function of the user role }\end{array}$ \\
\hline
\end{tabular}

The environment is an empty room where the user can move, with the 3D model in the middle of the scene. Figure 3 shows information from other models provided around the CAD model in a graph. Many factors motivated the choice of a graph visual metaphor. First, the data produced by the design of a system can be stored in this type of database (Bajaj and Hedberg, 2018). Second, this shape allows the user to visualise in a single view many elements of the models simultaneously, and graph exploration is more efficient using virtual reality than in a 2D screen (Kwon et al., 2016).
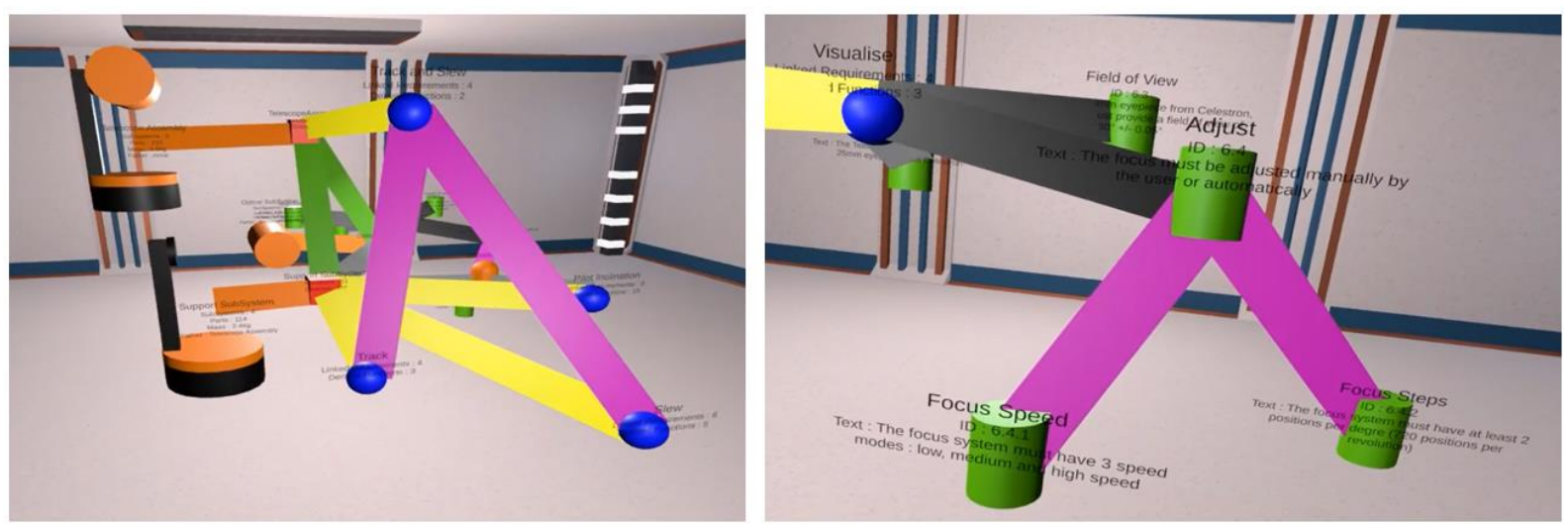

Figure 3. Visualising information from the architecture model around the CAD model.

\subsubsection{Interactions}

As for visualisation, we answer the three questions: What interaction we want with the 3D model or the elements of the graph, why we need these interactions, and how these interactions may be implemented in the environment. 


\section{Navigating in the 3D views}

Table 3. Specification of the 3D navigation interaction

\begin{tabular}{|l|l|l|}
\hline What? & Why? & How? \\
\hline $\begin{array}{l}\text { Interactions to navigate in the } \\
\text { different sub-assemblies or part } \\
\text { of the 3D model }\end{array}$ & $\begin{array}{l}\text { To select the assembly that the } \\
\text { user wants to study in particular }\end{array}$ & $\begin{array}{l}\text { Laser pointers to interact with } \\
\text { the 3D model }\end{array}$ \\
\cline { 2 - 3 } & $\begin{array}{l}\text { To analyse the sub-assemblies } \\
\text { decomposition of the system }\end{array}$ & $\begin{array}{l}\text { Clicking on an assembly to } \\
\text { decompose it }\end{array}$ \\
\hline
\end{tabular}

When clicking on the CAD model, a menu appears close to the user (Figure 4) with some information contained in the native CAD model. Thanks to this menu, clicking on the "decompose" button. we can decompose the model into its sub-systems. It is also possible to reach the father of the system, hide it, isolate it or study its equivalents in other models.

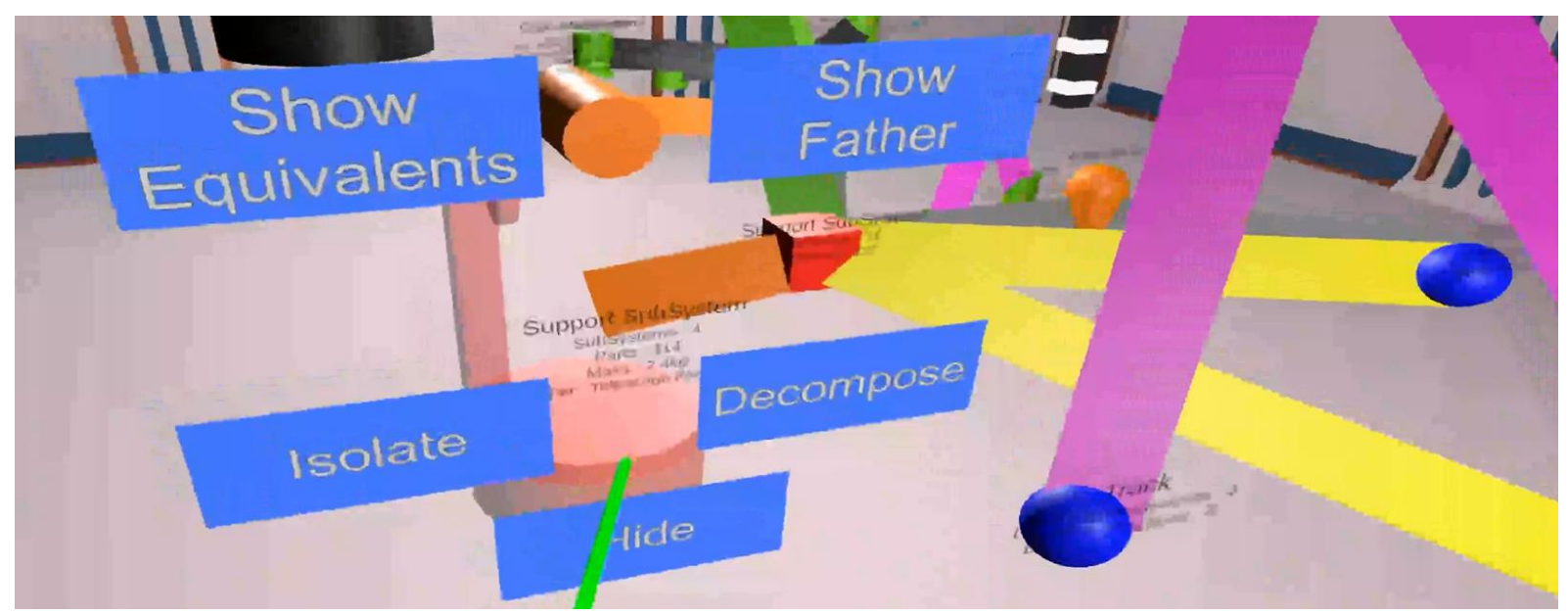

Figure 4. Interacting with the CAD model

Displaying information from architecture about the studied sub-system

Table 4. Specification of the information display interaction

\begin{tabular}{|l|l|l|}
\hline What? & Why? & How? \\
\hline $\begin{array}{l}\text { Researching information } \\
\text { about an assembly or sub- } \\
\text { assembly in the database }\end{array}$ & $\begin{array}{l}\text { To complete the CAD data with } \\
\text { architecture model data }\end{array}$ & $\begin{array}{l}\text { Choosing thanks to menu the } \\
\text { information the user wants to } \\
\text { visualise }\end{array}$ \\
\cline { 2 - 3 } & $\begin{array}{l}\text { To visualise in the same environment } \\
\text { data about an assembly or a part from } \\
\text { different models }\end{array}$ & $\begin{array}{l}\text { Display a list or a graph with } \\
\text { the data extracted from the } \\
\text { database }\end{array}$ \\
\hline
\end{tabular}

Hovering a node or any element on the scene with the laser pointer will display the name of this element, (Figure 5), for quick exploration.

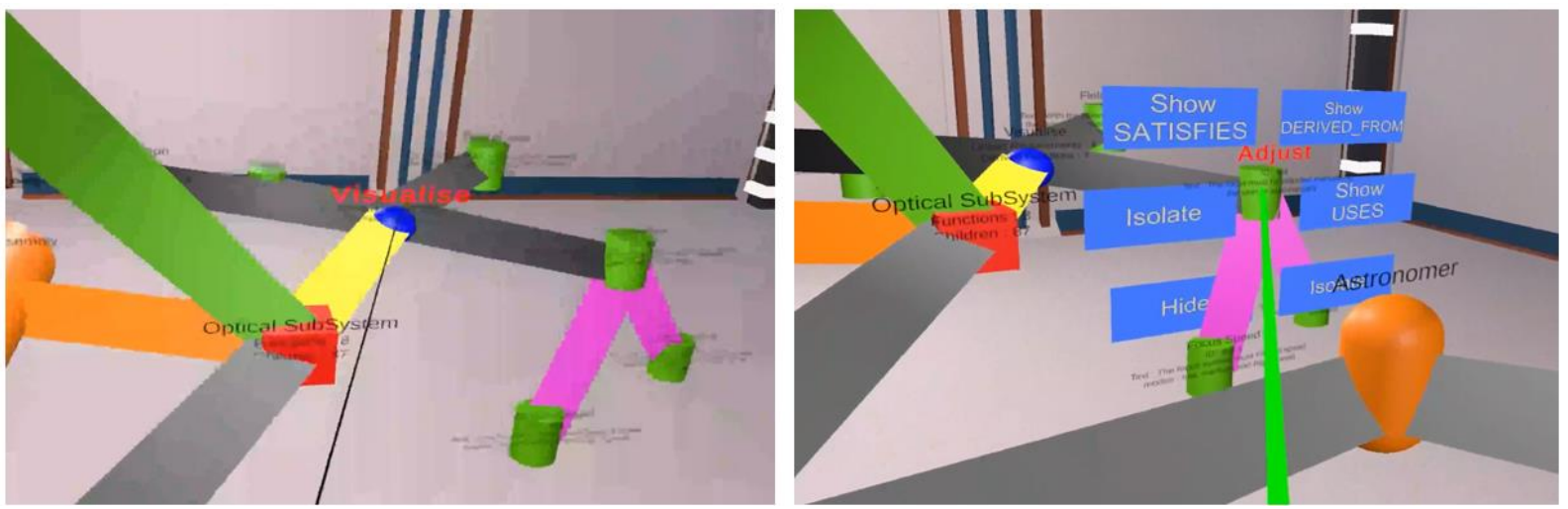

Figure 5. Interacting with nodes of the architecture graph data 
This preliminary version of the prototype enables us to learn several lessons. First, it is possible to unify multiple views of a model-centric design in a unique environment. Although during the first activities of the design process (specification, functional analysis, etc.), the 3D geometric model does not exist, there is a need to have 3D metaphors - e.g., a preliminary CAD design such as a skeleton - to locate data in space.

Although there are various ways to improve the current state of the prototype (adding models, adding other interactions, adding material to manage a design review, such as a checklist for example), the facility to navigate through different views without learning domain-specific languages and software is a first element to argue that:

\section{Answer to the Research Question:}

Yes, the unification of models in a unique and immersive virtual environment provides a holistic bird's-eye-view that facilitates a model-centric design review.

\section{DISCUSSION AND CONCLUSION}

The extensive use of models in a model centric-design approach is giving birth to new communication problems, especially during design reviews. Indeed, design reviews are mainly limited to 2D illustrations of models whereas 3D stereoscopy has demonstrated its benefit in several scenarios, such as the review of mechanical assemblies and software architecture. Thus, in this paper, we introduced an immersive virtual environment for collaboratively reviewing model-centric designs. In a unique virtual space, users can navigate through the different models through digital threads without the need to learn domain-specific languages and modelling software. Anyone can consider the view defined by colleagues leading to a holistic bird's-eye-view of the virtual definition of a system.

As previously discussed, this initial demonstrator showed that it facilitates the review of a modelcentric design, but it requires to be improved. First, we want to integrate more models than only the architecture and the 3D model. Safety models, multi-engineering simulation models, etc. will be integrated into the digital threads and the virtual environment. Another aspect to be improved is collaboration. We argue that such an environment will encourage communication among stakeholders, but this demonstrator is a single-user application. Using networking, we want to enable collaboration with several users. The visual metaphors shall also take more advantage of the unlimited virtual space. At last, the future goal is to validate our proposal with sound experiences before expending the tests of the virtual environment on other scenarios, as we did for verification activities (Romero, Pinquié and Noël, 2021).

\section{REFERENCES}

Aromaa, S. et al. (2012) 'Benefits of the use of virtual environments in product design review meeting', in Proceedings of International Design Conference, DESIGN, pp. 355-364.

Averbukh, V. et al. (2019) 'Metaphors for Software Visualization Systems Based on Virtual Reality', in Springer Nature Switzerland AG 2019 (ed.) Augmented Reality, Virtual Reality, and Computer Graphics. AVR 2019. Lecture Notes in Computer Science. Springer, Cham., pp. 60-70. https://dx.doi.org/10.1007/978-3030-25965-5_6.

Bajaj, M. and Hedberg, T. (2018) 'System Lifecycle Handler - Spinning a Digital Thread for Manufacturing', INCOSE International Symposium, 28(1), pp. 1636-1650. https://dx.doi.org/10.1002/j.2334-5837. 2018.00573.x.

Bennett, R., Zielinski, D. J. and Kopper, R. (2014) 'Comparison of interactive environments for the archaeological exploration of 3D landscape data', in 2014 IEEE VIS International Workshop on 3DVis (3DVis). IEEE, pp. 67-71. https://dx.doi.org/10.1109/3DVis.2014.7160103.

de Casenave, L. and Lugo, J. E. (2018) 'Effects of Immersion on Virtual Reality Prototype Design Reviews of Mechanical Assemblies', in Volume 7: 30th International Conference on Design Theory and Methodology. American Society of Mechanical Engineers, pp. 1-11. https://dx.doi.org/10.1115/DETC2018-85542.

Chandler, T. et al. (2015) 'Immersive Analytics'. Available at: https://ieeexplore.ieee.org/document/8698351/.

Chen, J., Zucco, G. and Olechowski, A. (2019) 'A Survey of Design Reviews: Understanding Differences by Designer-Roles and Phase of Development', Proceedings of the Design Society: International Conference on Engineering Design, 1(1), pp. 2745-2754. https://dx.doi.org/10.1017/dsi.2019.281.

Dai, X. and Velde, F. (2017) 'How Explicit Are We in a Design Meeting: Investigation on Meeting Knowledge', in Proceedings of the 21st International Conference on Engineering Design (ICED17). Vancouver, Canada, pp. 337-344. 
Hedberg, T. D. J. (2018) Enabling Connections in the Product Lifecycle using the Digital Thread. Virginia Polytechnic Institute and State University.

Herzog, E., Pandikow, A. and Ab, S. (2005) 'SysML - an Assessment'.

Huet, A. et al. (2020) 'Knowledge Graph of Design Rules for a Context-Aware Cognitive Design Assistant.', in Nyffenegger F., Ríos J., Rivest L., B. A. (eds) (ed.) Product Lifecycle Management Enabling Smart X. PLM 2020. https://dx.doi.org/10.1007/978-3-030-62807-9_27.

Kwon, O.-H. et al. (2016) 'A Study of Layout, Rendering, and Interaction Methods for Immersive Graph Visualization', IEEE Transactions on Visualization and Computer Graphics, 22(7), pp. 1802-1815. https://dx.doi.org/10.1109/TVCG.2016.2520921.

Louison, C. et al. (2017) 'Operators' accessibility studies for assembly and maintenance scenarios using virtual reality’, Fusion Engineering and Design. Elsevier B.V., 124, pp. 610-614. https://dx.doi.org/10.1016/j. fusengdes.2017.03.017.

Maletic, J., Leigh, J. and Marcus, A. (2001) 'Visualizing software in an immersive virtual reality environment', Proceedings of ICSE, p. 6. https://dx.doi.org/10.1016/j.procs.2014.07.020.

Mhenni, F. et al. (2014) 'A SysML-based methodology for mechatronic systems architectural design', Advanced Engineering Informatics. Elsevier Ltd, 28(3), pp. 218-231. https://dx.doi.org/10.1016/j.aei.2014.03.006.

Michalos, G. et al. (2018) 'Workplace analysis and design using virtual reality techniques', CIRP Annals. CIRP, 67(1), pp. 141-144. https://dx.doi.org/10.1016/j.cirp.2018.04.120.

Mirhosseini, K. et al. (2014) 'Benefits of 3D immersion for virtual colonoscopy', in 2014 IEEE VIS International Workshop on 3DVis (3DVis). IEEE, pp. 75-79. https://dx.doi.org/10.1109/3DVis.2014. 7160105.

Oberhauser, R. and Lecon, C. (2017) 'Immersed in Software Structures : A Virtual Reality Approach', in ACHI 2017 : The Tenth International Conference on Advances in Computer-Human Interactions Immersed, pp. $181-186$.

Romero, V., Pinquié, R. and Noël, F. (2021) 'A Computer-Aided Verification Process for Engineered Systems', in CIGI. Grenoble.

Schreiber, A. and Misiak, M. (2018) 'Visualizing Software Architectures in Virtual Reality with an Island Metaphor', in Virtual, Augmented and Mixed Reality: Interaction, Navigation, Visualization, Embodiment, and Simulation. VAMR 2018. Lecture Notes in Computer Science. Springer, Cham., pp. 168-182. https://dx.doi.org/10.1007/978-3-319-91581-4_13.

Waurich, V. and Weber, J. (2017) 'Interactive FMU-Based Visualization for an Early Design Experience', Proceedings of the 12th International Modelica Conference, Prague, Czech Republic, May 15-17, 2017, 132, pp. 879-885. https://dx.doi.org/10.3384/ecp17132879. 